\title{
Melatonin attenuates renal fibrosis in diabetic mice by activating the AMPK/PGC1 $\alpha$ signaling pathway and rescuing mitochondrial function
}

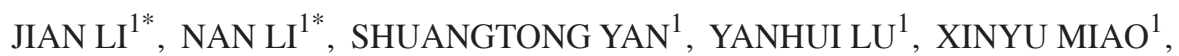 \\ ZHAOYAN GU ${ }^{1}$ and YINGHONG SHAO ${ }^{2}$ \\ ${ }^{1}$ Department of Geriatric Endocrinology, Chinese PLA General Hospital, National Clinical Center of Geriatric Medicine; \\ ${ }^{2}$ Outpatient Department, Chinese PLA General Hospital, Beijing 100853, P.R. China
}

Received February 17, 2018; Accepted August 30, 2018

DOI: $10.3892 / \mathrm{mmr} .2018 .9708$

\begin{abstract}
Mitochondrial homeostasis is a highly regulated process that serves a critical role in the maintenance of renal structure and function. The growing interest in the field of mitochondrial homeostasis promises to provide more information regarding the mechanisms involved in diabetic renal fibrosis, and aid in the development of novel strategies to combat the disease. In the present study, the effects of melatonin on renal damage in mice with diabetes were evaluated and the underlying mechanisms were investigated. Cellular apoptosis was determined using TUNEL assay and western blotting. Mitochondrial function was measured using fluorescence assay and western blotting. The results indicated that diabetic renal fibrosis was associated with 5'adenosine monophosphate-activated protein kinase (AMPK) downregulation. However, melatonin administration rescued AMPK activity, reduced diabetic renal fibrosis, alleviated glomerular apoptosis and preserved kidney function. The functional experiments demonstrated that melatonin-induced AMPK activation enhanced peroxisome proliferator-activated receptor $\gamma$ coactivator 1- $\alpha(\mathrm{PGC} 1 \alpha)$ expression, sustained mitochondrial function and blocked mitochondrial apoptosis, leading to protection of the glomerulus against glucotoxicity. However, loss of AMPK and PGC1 $\alpha$ negated the protective effects of melatonin on mitochondrial homeostasis, glomerular survival and diabetic renal fibrosis. In summary, the present study revealed that melatonin rescued impaired mitochondrial function and reduced glomerular apoptosis in the context
\end{abstract}

Correspondence to: Dr Yinghong Shao, Outpatient Department, Chinese PLA General Hospital, 28 Fuxing Road, Beijing 100853, P.R. China

E-mail: shaoyh021206@sina.com

${ }^{*}$ Contributed equally

Key words: diabetic renal dysfunction, melatonin, AMPK, PGC1 $\alpha$, mitochondrial homeostasis of diabetic renal fibrosis by activating the AMPK/PGC1 $\alpha$ pathway.

\section{Introduction}

Diet-induced diabetes is one of the leading causes of mortality worldwide. The global prevalence of diabetes is estimated to affect 439 million adults (aged 20-79 years) by 2030 (1). Between 2010 and 2030, it has been predicted that there will be a $69 \%$ increase in the number of patients with diabetes in developing countries (2). Notably, it has been proposed that $40 \%$ of patients with type 2 diabetes mellitus may develop renal fibrosis, which is a major cause of end-stage renal disease. However, current treatment strategies for renal fibrosis are the same in diabetic patients as in non-diabetic patients, and they do not address the underlying causes of renal dysfunction.

Mitochondria are the energy centers of the cell and have been reported to serve a critical role in regulating kidney function (3). Increasing evidence suggests that kidney complications associated with diabetes converge on mitochondria as an epicenter for diabetes-induced renal fibrosis (4). The hallmarks of renal fibrosis include glomerular cell apoptosis due to high glucose-induced stress, and renal interstitial fibrosis caused by accumulation of the extracellular matrix, which contributes to the irreversible decline in renal function. Notably, mitochondrial injury is observed under all of these situations. Mitochondria are particularly susceptible to diabetic insults and, at the molecular level, damaged mitochondria produce excessive reactive oxygen species (ROS), release pro-apoptotic factors into the cytoplasm, impair cellular energy metabolism and activate caspase-9-dependent apoptotic signaling (5-8), thus augmenting renal injury and promoting kidney fibrosis. Therefore, protecting mitochondrial function and preventing mitochondria-initiated glomerular apoptosis may delay the development of diabetes-associated renal fibrosis.

Recently, several studies have documented the involvement of melatonin in kidney protection (9). Melatonin presumably enters mitochondria through melatonin receptor-dependent and -independent manners $(10,11)$. Measurement of the sub-cellular distribution of melatonin revealed that the concentration of melatonin in mitochondria greatly 
exceeds that in blood (12), thus suggesting that melatonin may specifically target mitochondria. In addition, there is accumulating evidence demonstrating the protective role of melatonin in diabetes-associated renal fibrosis and glomerular apoptosis $(13,14)$. Melatonin can inhibit nicotinamide-adenine dinucleotide oxidase activity (15), reduce Raf-1 proto-oncogene, serine/threonine kinase/extracellular signal-regulated kinases signaling (16), activate the cyclic guanosine 3',5'-monophosphate-protein kinase $\mathrm{G}$ axis (17) and suppress the nuclear receptor subfamily 4 group A member 1/DNA-dependent protein kinase, catalytic subunit/tumor protein p53 (p53) cascade (18), thus favoring the survival of glomeruli and inhibiting the progression of diabetes-induced renal fibrosis. Melatonin has also been reported to protect mitochondrial function and structure in the context of high glucose stimulus $(19,20)$. However, it remains unclear as to whether melatonin has the ability to preserve mitochondrial homeostasis during the development of diabetic renal fibrosis and, if so, what molecular mechanisms link melatonin to mitochondrial protection under high glucose attack.

It has previously been suggested that 5'adenosine monophosphate-activated protein kinase (AMPK) signaling is closely associated with diabetic renal dysfunction (20). AMPK, a sensor of cellular energy production, is capable of regulating mitochondrial dynamics and controlling mitophagy (21-24). Furthermore, several studies have identified melatonin as the upstream trigger of AMPK activation $(19,25)$. However, whether AMPK is required for the protective role of melatonin in mitochondrial damage upon hyperglycemia exposure remains unclear. The aim of the present study was to investigate the effects of melatonin on mitochondrial homeostasis and kidney fibrosis under diabetic stress, as well as to understand the underlying mechanisms.

\section{Materials and methods}

Animal and cellular experiments. Male wild-type (WT) mice $(\mathrm{n}=60$, weight, $250 \pm 10 \mathrm{~g})$ and AMPK knockout $\left(\mathrm{AMPK}^{-/}\right)$ mice ( $\mathrm{n}=60$, weight, $250 \pm 10 \mathrm{~g}$ ) were obtained from Jackson Laboratory (Bar Harbor, ME, USA). All mice were housed under standard laboratory conditions $\left(27^{\circ} \mathrm{C}, 40-60 \%\right.$ humidity, 12-h light/dark cycle) with free access to water and on a standard laboratory diet. Eight-week-old WT and $\mathrm{AMPK}^{-1}$ mice were injected with streptozotocin (STZ; $50 \mathrm{mg} / \mathrm{kg} /$ day) for 5 days (20). Subsequently, melatonin $(20 \mathrm{mg} / \mathrm{kg} / \mathrm{day}$; Sigma-Aldrich; Merck KGaA, Darmstadt, Germany) was administered intraperitoneally into $\mathrm{WT}$ and $\mathrm{AMPK}^{-/}$mice over the course of 12 weeks. The animal experiments were performed for a 12-week period ( $n=6 /$ group). WT mice injected with PBS were termed the control group; WT mice injected with STZ were termed the STZ+WT group; WT mice treated with STZ and melatonin were termed the Mel+STZ+WT group; and, $\mathrm{AMPK}^{-/-}$mice treated with $\mathrm{STZ}$ and melatonin were termed the Mel+STZ+AMPK ${ }^{-/}$group. At the end of the experiment, blood pressure was measured in conscious, acclimated mice using the tail-cuff method. Kidney tissues were harvested following the sacrifice of the mice. A part of the kidney was snap-frozen in liquid nitrogen and the other part was fixed with $10 \%$ PBS-buffered formalin, processed and embedded in paraffin. All experimental protocols were approved by the Ethics Committee of Chinese PLA General Hospital (Beijing, China).

The rat glomerular mesangial cell line, HBZY-1, was purchased from the Cell Bank of the Chinese Academy of Sciences (Shanghai, China). Cells were cultured according to the supplier's protocols in RPMI medium (Thermo Fisher Scientific, Inc., Waltham, MA, USA) supplemented with $10 \%$ fetal bovine serum (HyClone; GE Healthcare Life Sciences, Logan, UT, USA) at $37^{\circ} \mathrm{C}$ in $5 \% \mathrm{CO}_{2}$. To mimic high glucose damage, cells were cultured in high glucose medium $(25 \mathrm{mmol} / \mathrm{l})$, as opposed to normal glucose medium (5.5 mmol/l), for $\sim 12 \mathrm{~h}$ according to a previous study (26). The cells were incubated with $5 \mu \mathrm{M}$ melatonin for $\sim 12 \mathrm{~h}$ before high-glucose stress (18).

Sample preparation and histological analysis. The kidneys were harvested, fixed for $1 \mathrm{~h}$ in $4 \%$ (w/v) paraformaldehyde at room temperature, and rapidly frozen in Optimal Cutting Temperature Compound (Agar Scientific, Ltd., Stansted, $\mathrm{UK}$ ) for the preparation of cryosections (size, $4 \mu \mathrm{m})(27)$. Hematoxylin and eosin (H\&E), Masson's trichrome (cat. no. KGMST-8004; Nanjing KeyGen Biotech Co., Ltd., Nanjing, China) and periodic acid Schiff-methenamine (PASM; cat. no. 395B; Sigma-Aldrich; Merck KGaA) staining were performed at room temperature, following which, samples were observed under an inverted microscope (magnification, x40; BX51; Olympus Corporation, Tokyo, Japan).

ELISA. Blood samples from tail vein were collected before the mice were sacrificed. Then, laminin (Mouse Laminin ELISA kit; cat. no. ab119572; Abcam, Cambridge, UK) and pre-collagen III (Mouse Collagen Type III ELISA kit; cat. no. MBS727234; MyBiosource, San Diego, CA, USA) in blood samples were measured via ELISA analysis, according to a previous study (28). Blood urea nitrogen (BUN; Mouse Blood Urea Nitrogen ELISA kit; cat. no. MBS751125; MyBiosource) and serum creatinine levels (Creatinine Assay kit; cat. no. ab65340; Abcam) were determined via ELISA based on the manufacturers' protocols.

Measurement of glucose levels and urinary albumin. After $12 \mathrm{~h}$ of fasting, the glucose levels in venous blood samples drawn from the tail vein were measured using a glucometer (Roche Diagnostics GmbH, Mannheim, Germany). Urine samples were collected prior to the sacrifice of the mice (29). To collect morning spot urine samples, animals were placed in metabolic cages at the beginning of the light cycle and were kept for $2 \mathrm{~h}$ with access to water, but not food. To obtain 24-h urine samples, animals were placed in metabolic cages at the beginning of the light cycle and were kept for $24 \mathrm{~h}$ with free access to water and on a standard laboratory diet (30). Urinary albumin content (Mouse Albumin ELISA kit; cat. no. ab108792; Abcam,) was determined according to the manufacturer's protocols.

Immunofluorescence staining. The cells were first washed in cold PBS, permeabilized with $0.1 \%$ Triton-X-100 for $10 \mathrm{~min}$ at $4^{\circ} \mathrm{C}$ and blocked with $10 \%$ goat serum albumin (Invitrogen; Thermo Fisher Scientific, Inc.) for $1 \mathrm{~h}$ at room temperature. Subsequently, samples were incubated with cytochrome 
c(cyt-c) antibody (1:1,000; cat. no. ab133504; Abcam) and cleaved caspase-3 antibody (1:1,000; cat. no. 9664; Cell Signaling Technology, Inc. Danvers, MA, USA), overnight at $4^{\circ} \mathrm{C}$ (31). Following three washes in PBS, Alexa-Fluor 116488 donkey anti-rabbit secondary antibody $(1: 1,000$; cat.no. A-21206; Invitrogen; Thermo Fisher Scientific, Inc.) was added to the samples for $1 \mathrm{~h}$ at room temperature (32). Images were observed with an inverted fluorescence microscope (magnification, x40; BX51; Olympus Corporation).

Western blotting. The proteins isolated from kidney tissues with the help of RIPA buffer (Thermo Fisher Scientific, Inc.) were from three mice in each group. Proteins were also isolated from cells using RIPA buffer (Thermo Fisher Scientific, Inc.). The protein concentration was analyzed using the bicinchoninic acid protein assay (Thermo Fisher Scientific, Inc.). In the present study, a total of $40 \mu \mathrm{g}$ cell proteins and $60 \mu \mathrm{g}$ tissue proteins were separated by $12-15 \%$ SDS-PAGE. Following electrophoresis, the proteins were transferred onto a polyvinylidene fluoride membrane (Roche Applied Science, Penzberg, Germany). The membranes were subsequently blocked with 5\% non-fat milk for $1 \mathrm{~h}$ at room temperature prior to incubation with the primary antibodies. The primary antibodies used were: Pro-caspase-3 (1:1,000; cat. no. 9662; Cell Signaling Technology, Inc.), cleaved caspase-3 (1:1,000; cat. no. 9664; Cell Signaling Technology, Inc.), cellular inhibitor of apoptosis protein 1 (c-IAP; 1:1,000; cat. no. 4952; Cell Signaling Technology, Inc.), B-cell lymphoma 2 (Bcl2; 1:1,000, cat. no. 3498; Cell Signaling Technology, Inc.), caspase-9 (1:1,000; cat. no. ab32539; Abcam), transforming growth factor (TGF) $\beta(1: 1,000$; cat. no. 3711 ; Cell Signaling Technology, Inc.), matrix metalloproteinase 9 (MMP9; 1:1,000; cat. no. 13667; Cell Signaling Technology, Inc.), cyclophilin D (1:1,000; cat. no. ab181983; Abcam), p-cyclophilin D antibody (1:1,000, bioss, cat. no. bs-9878R), collagen I (1:1,000; cat. no. ab34710; Abcam), collagen III (1:1,000; cat. no. ab7778; Abcam), Bcl-2-associated X protein (Bax; 1:1,000; cat. no. ab32503; Abcam), AMPK (1:1,000; cat. no. ab131512; Abcam), phosphorylated (p)-AMPK (1:1,000, cat. no. ab23875; Abcam) and peroxisome proliferator-activated receptor $\gamma$ coactivator 1- $\alpha$ (PGC1 $\alpha$; 1:1,000; cat. no. 2178; Cell Signaling Technology, Inc.) The secondary antibodies used in the present study were: Horseradish peroxidase (HRP)-coupled secondary antibodies (1:2,000; cat. nos. 7074 and 7076; Cell Signaling Technology, Inc.). Band intensities were normalized to the respective $\beta$-actin (1:2,000; cat. no. ab8224; Abcam) and/or GAPDH (1:1,000, cat. no. 5174; Cell Signaling Technology, Inc.) internal control signal intensity with the help of Quantity One Software (version 4.6.2; Bio-Rad Laboratories, Inc., Hercules, CA,USA) (29). Bands were detected using an enhanced chemiluminescence substrate (Applygen Technologies, Inc., Beijing, China). The experiment was repeated three times (33).

RNA isolation and reverse transcription-quantitative polymerase chain reaction $(R T-q P C R)$. TRIzol ${ }^{\circledR}$ reagent (Invitrogen; Thermo Fisher Scientific, Inc.) was used to isolate total RNA from tissues (34). Subsequently, the Reverse Transcription kit (Kaneka Eurogentec S.A., Seraing, Belgium) was applied to transcribe RNA ( $1 \mu \mathrm{g} / \mathrm{group})$ into cDNA according to the manufacturer's protocol. RT-qPCR was performed with primers and matched probes from the Universal Fluorescence-labeled
Probe Library (Roche Diagnostics, Basel, Switzerland) using SYBR $^{\mathrm{TM}}$ Green PCR Master Mix (Thermo Fisher Scientific, Inc.) (35). The primers used in the present study were: Bax forward, 5'-GTCCTATTCTGATGGATCCC-3' and reverse, 5'-GCTACGGTACCATGGCCTACG-3'; Bad, 5'-CCTAGA TTCAGTGACTGAG-3' and reverse, 5'-ACCTAACCGATG GCGGTCGAGTGC-3'; Survivin, 5'-ATCCGTTGTCCA GTCTTAGTCTA-3' and reverse, 5'-GGTCGGTAGATTCAT TAATGAT-3' and GAPDH forward, 5'-GCGGATGAACGT GGACGTGAC -3' and reverse, 5'-AACGTGGTCCAGACC AATGCG-3'). The cycling conditions were: $95^{\circ} \mathrm{C}$ for $8 \mathrm{~min}$, followed by 35 cycles of $95^{\circ} \mathrm{C}$ for $10 \mathrm{sec}$ and $72^{\circ} \mathrm{C}$ for $12 \mathrm{sec}$, for telomere PCR. The mRNA ratio of the target genes to GAPDH was calculated using the $2^{-\Delta \Delta \mathrm{Cq}}$ method (36).

Electron transport chain complex (ETC) activity detection. Electron transport chain complex I activity (Electron transport chain Complex I Assay kit; cat.no. MBS2540528; MyBiosource), Electron transport chain complex II activity (Complex II Enzyme Activity Microplate Assay kit; cat. no. ab109908; Abcam,) and Electron transport chain complex V activities (MitoTox ${ }^{\mathrm{TM}}$ Complex V OXPHOS Activity Assay kit; cat. no. ab109907; Abcam) were determined according to the manufacturers' protocols.

Cellular ROS detection. To observe cellular ROS levels, the dihydroethidium ROS probe (Molecular Probes; Thermo Fisher Scientific, Inc.) was incubated with the cells $\left(1 \times 10^{5}\right)$ for $\sim 30 \mathrm{~min}$ at $37^{\circ} \mathrm{C}$ in the dark. Subsequently, the cells were washed with PBS to remove the ROS probe. The cells were immediately analyzed under a fluorescence microscope (37). Image-Pro Plus version 6.0 (Media Cybernetics, Rockville, MD, USA) was used to obtain the fluorescence densities, which were normalized to that of the control group.

Mitochondrial permeability transition pore (mPTP) opening assay, JC-1 staining and ATP production. mPTP opening is an early event in mitochondrial apoptosis. In the present study, mPTP opening was measured via tetramethylrhodamine ethyl ester (TMRE) fluorescence. Samples were washed three times with PBS and were then stained with TMRE dye (38). The baseline fluorescence of TMRE was recorded and, after $30 \mathrm{~min}$, TMRE fluorescence was recorded again. Image-Pro Plus version 6.0 (Media Cybernetics) was used to obtain the fluorescence densities of TRME fluorescence. According to a previous study (39), the mPTP opening rate was determined as the time for the fluorescence intensity to decrease by half of the baseline fluorescence intensity.

Mitochondrial potential was assessed using a JC-1 probe, a sensitive fluorescent dye used to detect alterations in mitochondrial potential (34). Following treatment, cells $\left(1 \times 10^{5}\right)$ were incubated with $10 \mathrm{mg} / \mathrm{ml} \mathrm{JC}-1$ for $10 \mathrm{~min}$ at $37^{\circ} \mathrm{C}$ in the dark and monitored with a fluorescence microscope (magnification, x100; BX51; Olympus Corporation). Red fluorescence was attributable to potential-dependent dye aggregation in the mitochondria. Green fluorescence, reflecting the monomeric form of JC-1, appeared in the cytosol following mitochondrial membrane depolarization.

ATP production was detected to monitor mitochondrial function. Firstly, the samples were washed three times with cold 
PBS. The samples were then lysed using the RIPA Lysis Buffer (Beyotime, China, cat. No:P0013E) and the Luciferase-based ATP Assay kit (Beyotime Institute of Biotechnology, Haimen, China) was used according to a previous study (25). ATP production was measured using a microplate reader (40).

Lactate dehydrogenase ( $\mathrm{LDH}$ ) assay and caspase-3/9 activity detection. LDH is released into the cell culture supernatant when cell membranes rupture $(36,41)$. To evaluate LDH levels (cells density $1 \times 10^{5}$ ), an LDH Release Detection kit (Beyotime Institute of Biotechnology) was used according to the manufacturer's protocol.

To analyze changes in caspase-3/9, caspase-3/9 activity kits (Beyotime Institute of Biotechnology) were used, according to the manufacturer's protocols (42). To analyze caspase-3 activity, $5 \mu 14 \mathrm{mM}$ DEVD-pNA substrate (final concentration, $200 \mu \mathrm{M})$ was added to the cells $\left(1 \times 10^{5}\right)$ for $2 \mathrm{~h}$ at $37^{\circ} \mathrm{C}$. To measure caspase-9 activity, $5 \mu 14$ mM LEHD-p-NA substrate (final concentration, $200 \mu \mathrm{M}$ ) was added to the samples for $1 \mathrm{~h}$ at $37^{\circ} \mathrm{C}$. Subsequently, caspase activity was quantified by spectrophotometric detection at $400 \mathrm{~nm}$ using a microplate reader (43).

MTT and terminal deoxynucleotidyl-transferase-mediated dUTP nick end labeling (TUNEL) assays. MTT experiments were performed in 96-well plates to analyze cellular viability. Samples $\left(1 \times 10^{5}\right)$ were washed three times with PBS and $50 \mu 1$ MTT reagent was added to each well. The samples were incubated for $4 \mathrm{~h}$ at $37^{\circ} \mathrm{C}$ in a humidified atmosphere containing $5 \% \mathrm{CO}_{2}$. Subsequently, the MTT solution was removed, $200 \mu 1$ dimethyl sulfoxide was added and the samples were incubated for $10 \mathrm{~min}$ at room temperature. Following the addition of Sorensen's buffer, the absorbance at $570 \mathrm{~nm}$ was measured (44).

To detect cell death, a TUNEL assay was performed using an In Situ Cell Death Detection kit (Roche Diagnostics $\mathrm{GmbH}$ ), according to the manufacturer's protocol. DAPI was used to label the nuclei at room temperature for $\sim 30 \mathrm{~min}$ and the cells were observed under an inverted fluorescence microscope (magnification, x40; BX51; Olympus Corporation) (45).

RNA silencing assay. To inhibit the expression of AMPK and PGC1 $\alpha$, small interfering (si)RNAs against AMPK and PGCl $\alpha$ were used to knockdown their expression. The siRNA against AMPK and PGC1 $\alpha$ as well as the negative control siRNA were purchased from Yangzhou Ruibo Biotech Co., Ltd (Yangzhou, China) (46). The siRNA sequences were as follows: AMPK siRNA sense strand, 5'-GCTTACUGA CTGACGT-3' and antisense strand, 3'-AUGUUACCGUAT TC-5'; PGC1 $\alpha$ siRNA sense strand, 5'-GUAGGUACTACC TA-3' and antisense strand, 3'-TUAUUTAGTTAACTGAT-5'; negative control siRNA sense strand, 5'-UUACCUTUCCAT GATGCT' and antisense strand, 3'-AUGTUGAAGUTC CGT-5'. To transfect siRNA into HBZY-1 cells, Opti-Minimal Essential Medium (Invitrogen; Thermo Fisher Scientific, Inc.) without serum or antibiotics was used to incubate cells for 24 h. Lipofectamine ${ }^{\circledR} 2000$ transfection reagent (Invitrogen; Thermo Fisher Scientific, Inc.) was added into the medium according to the manufacturer's protocol (47). Subsequently, siRNAs (70 nM/well of siRNA) were added into serum-free medium and incubated with cells for $72 \mathrm{~h}$. Cells were collected and proteins were extracted (48).

Statistical analysis. All data are expressed as the means \pm standard deviation and experiments were repeated $\geq 3$ times in the present study. Statistical analyses were performed with SPSS software version 17.0 (SPSS, Inc., Chicago, IL, USA). Statistical analysis was performed using one-way analysis of variance followed by Tukey's post hoc test. $\mathrm{P}<0.05$ was considered to indicate a statistically significant difference.

\section{Results}

Melatonin reduces diabetic renal fibrosis by activating $A M P K$. To verify the role of AMPK in diabetic renal fibrosis, western blotting was used to analyze AMPK expression. Compared with the control WT mice, STZ treatment significantly impaired AMPK activation; however, this effect was reversed by melatonin treatment (Fig. 1A and B). Furthermore, to confirm whether AMPK is necessary for melatonin-induced kidney protection under diabetic stress, $\mathrm{AMPK}^{-/-}$mice were used. As shown in Fig. 1C, renal fibrosis was observed using Masson's trichrome staining. Compared with the control WT mice, STZ-treated mice presented with severe kidney fibrosis and this change was partially reversed by melatonin treatment. However, AMPK deletion inhibited the protective effects of melatonin on renal fibrosis. In addition, the expression levels of collagen I and collagen III were assessed in kidney samples. Chronic hyperglycemia significantly enhanced collagen I and collagen III expression (Fig. 1D-F). Notably, this effect was inhibited by melatonin, whereas absence of AMPK completely abrogated the inhibitory effects of melatonin on collagen accumulation.

Furthermore, signaling factors associated with fibrosis, including TGF $\beta$ and MMP9, were more abundant in the kidney tissues of STZ-treated mice compared with the control WT mice (Fig. 1D, G and H). Treatment with melatonin significantly reduced TGF $\beta$ and MMP9 expression levels; however, the effects of melatonin were abolished by AMPK deletion. Furthermore, the serum concentrations of laminin and pre-collagen III were notably higher in STZ-treated mice compared with in control WT mice (Fig 1I and J). As expected, the high serum concentrations of laminin and pre-collagen III were significantly reduced by melatonin supplementation, whereas the effects of melatonin were reversed by AMPK deletion. Taken together, these results indicated that melatonin regulated diabetic renal interstitial fibrosis by activating AMPK.

Melatonin promotes glomerular survival in an $A M P K$-dependent manner. Glomerular apoptosis is the primary factor associated with the development of diabetic renal fibrosis; therefore, the role of melatonin and AMPK in glomerular apoptosis was assessed. Firstly, caspase-3 staining demonstrated that the renal tissue in STZ-treated mice exhibited increased cleaved caspase-3 expression compared with WT mice (Fig. 2A and B). However, melatonin administration reduced caspase- 3 expression, whereas the effects of melatonin were suppressed in response to AMPK deficiency. Similar results were obtained for the caspase-3 activity assay 

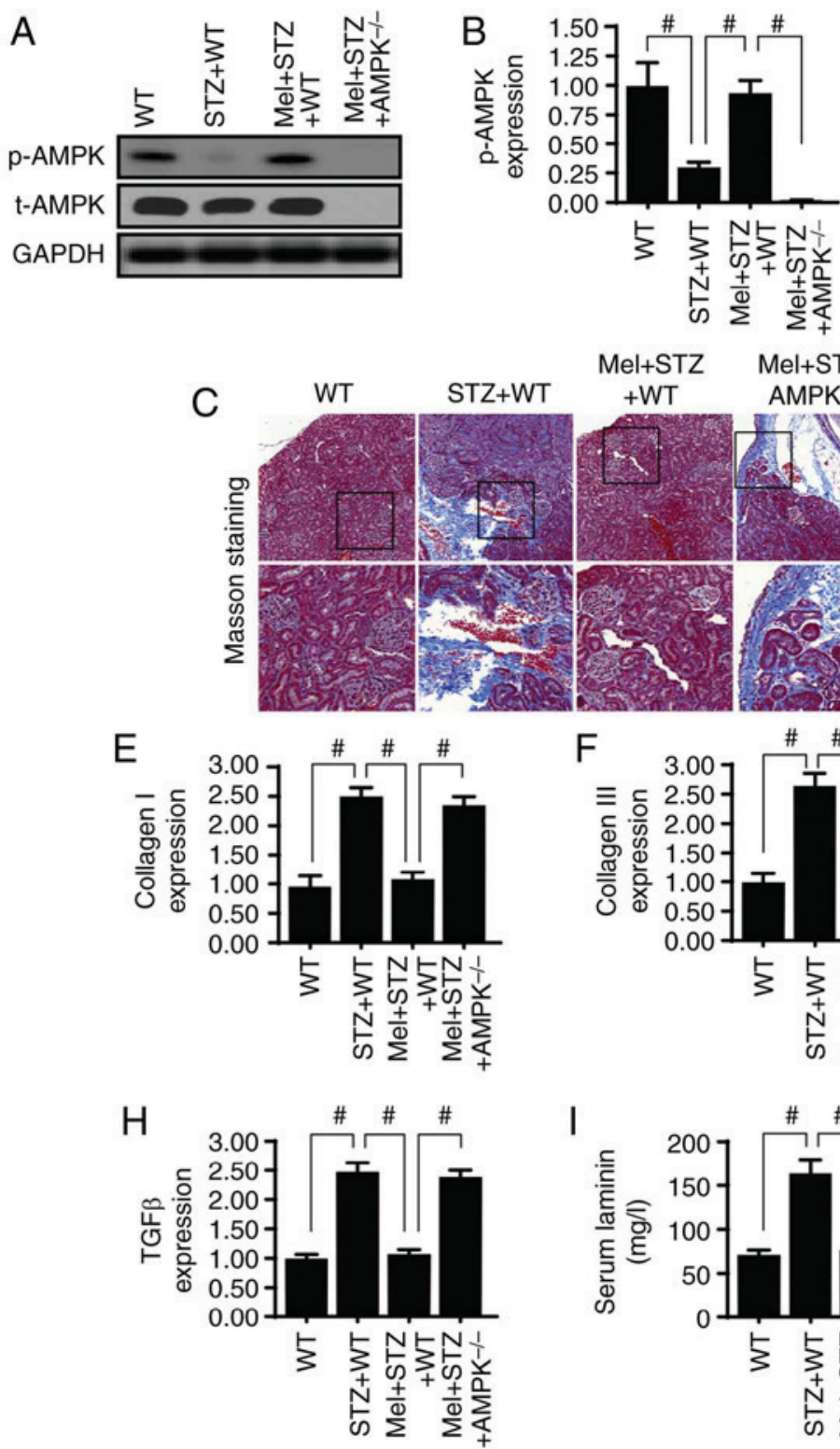
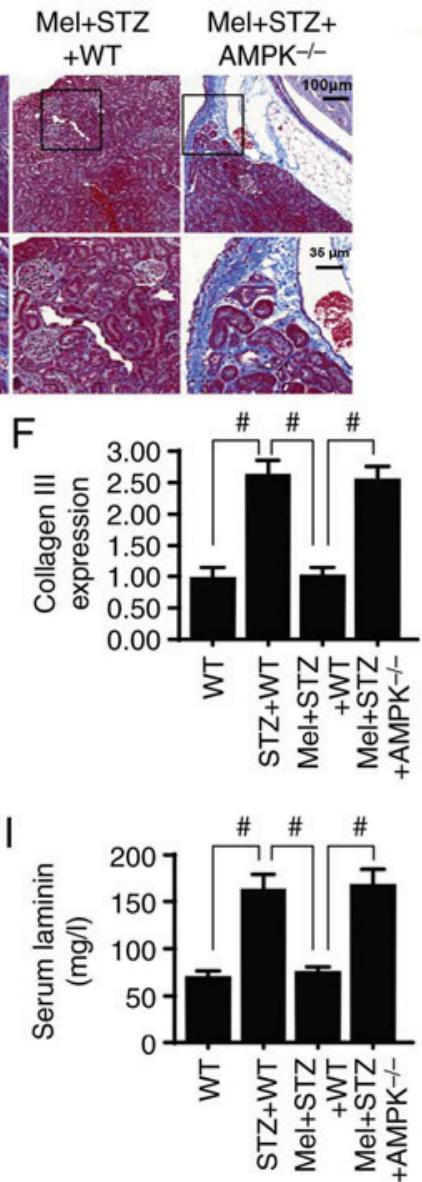
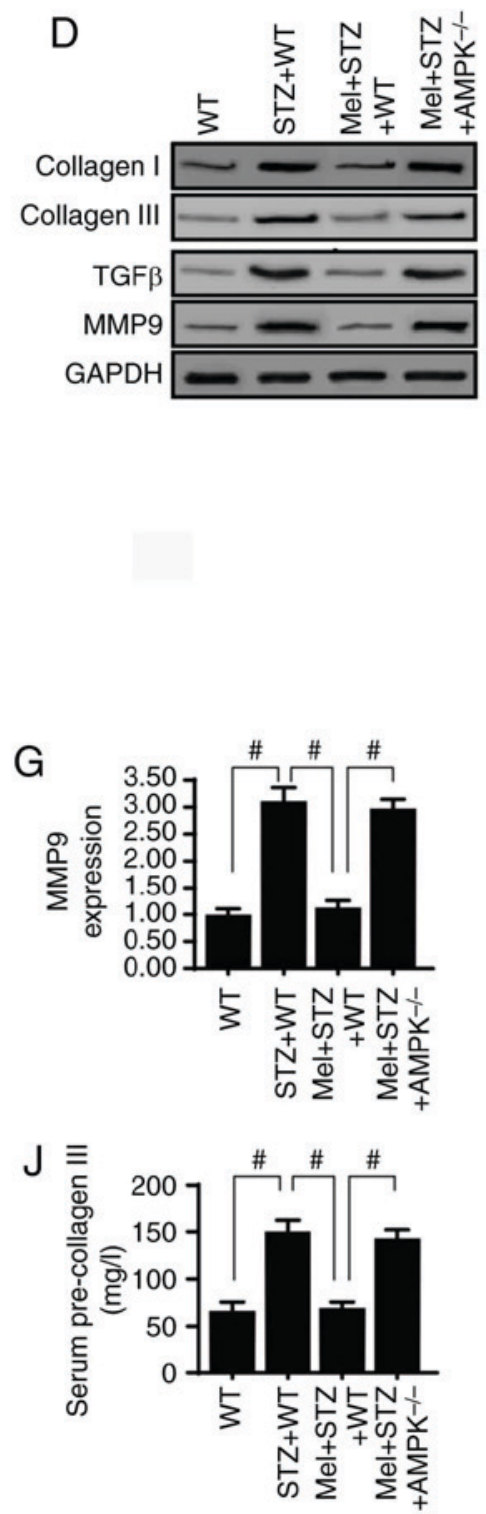

Figure 1. Melatonin reduces diabetic renal fibrosis via AMPK. (A and B) Western blot analysis of AMPK expression in the kidney tissues of STZ-treated mice in the presence or absence of melatonin. (C) Histological evaluation of kidney fibrosis (blue) using Masson's trichrome stain. (D-H) Western blot analysis of renal fibrosis-associated proteins. (I and J) Serum concentrations of laminin and pre-collagen III were determined by ELISA. "P<0.05. AMPK, 5'adenosine monophosphate-activated protein kinase; Mel, melatonin; MMP9, matrix metalloproteinase 9; p, phosphorylated; STZ, streptozotocin; t, total; TGF $\beta$, transforming growth factor $\beta$; WT, wild-type.

(Fig. 2C). Additionally, to investigate the protective role of melatonin and AMPK in glomerular apoptosis, western blotting was performed. Melatonin reduced the expression levels of pro-apoptotic proteins (caspase-3, -9 and Bax), but increased the expression of an anti-apoptotic factor (c-IAP), in an AMPK-dependent manner (Fig. 2D-H). Alterations in the expression levels of apoptosis-associated genes were also measured (Fig. 2I-L). The results demonstrated that the mRNA expression levels of $\mathrm{Bcl} 2$ and survivin were significantly reduced in the kidney tissues of STZ-treated mice compared with in the control WT mice. Conversely, Bax and $\mathrm{Bcl} 2$-associated agonist of cell death (Bad) mRNA expression levels were increased in STZ-treated mice compared with in control WT mice. Treatment with melatonin significantly reduced $\mathrm{Bad}$ and p53 mRNA expression, and increased $\mathrm{Bcl} 2$ and survivin mRNA expression in STZ-treated mice in an AMPK-dependent manner.

Melatonin ameliorates structural and functional renal damage in diabetes by upregulating AMPK. Diabetic kidney fibrosis is associated with renal hypertrophy. To evaluate renal hypertrophy, the mice were sacrificed and kidneys were weighed. Compared with the control WT mice, the kidneys of STZ-treated mice weighed more; however, this alteration was reversed by melatonin treatment (Fig. 3A). Loss of AMPK abolished the protective role of melatonin in kidney hypertrophy. These findings were further supported by the histopathological examination of H\&E- and PASM-stained kidney tissues. The results revealed that STZ-treated mice exhibited moderate glomeruli atrophy and fragmentation, 

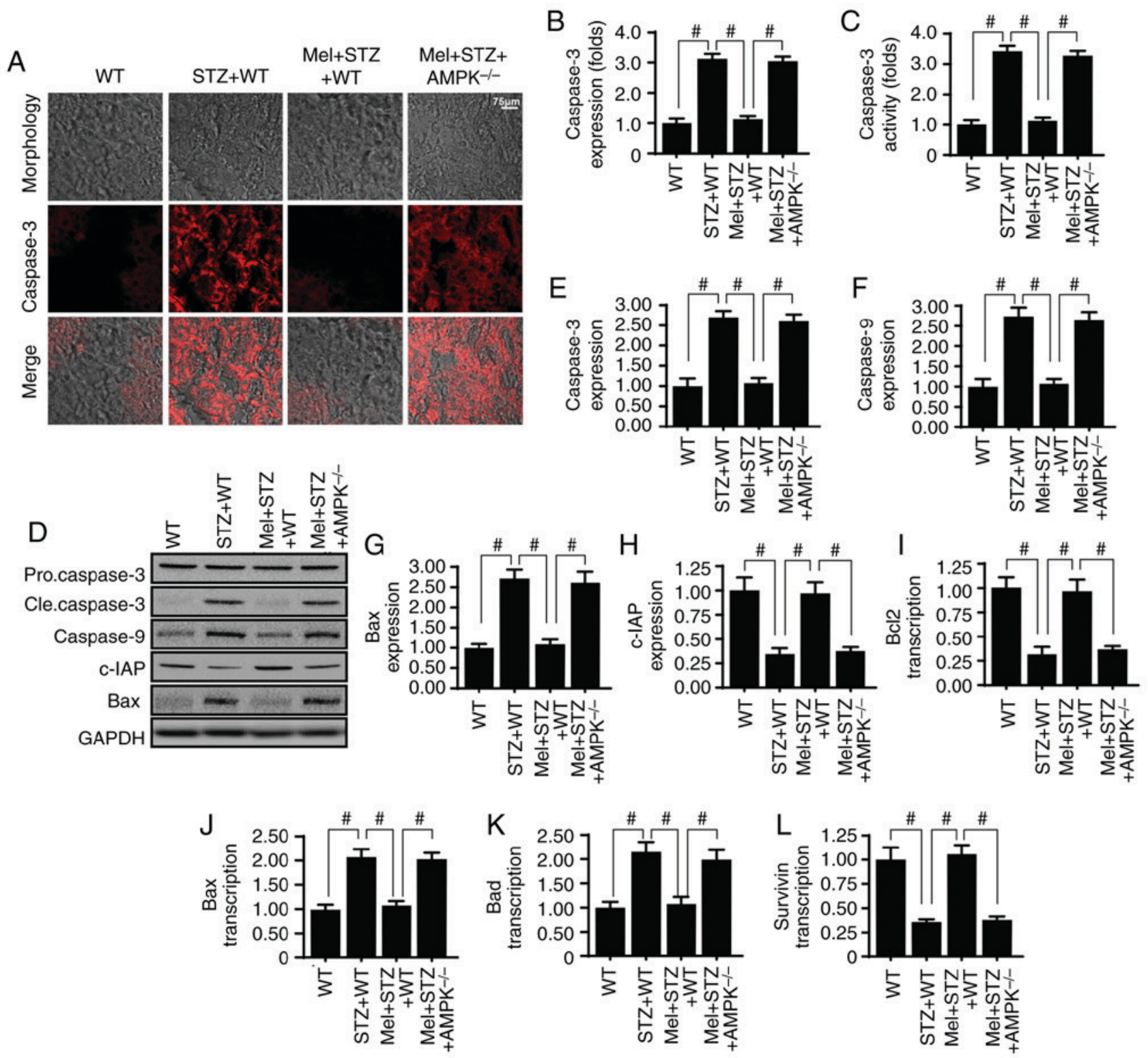

Figure 2. Melatonin activates AMPK and alleviates high glucose-induced glomerular apoptosis. (A and B) Caspase-3 expression in the kidney tissues of STZ-treated mice, in the presence or absence of melatonin. (C) Measurement of caspase-3 activity. (D-H) Western blot analysis of apoptosis-associated proteins . (I-L) Reverse transcription-quantitative polymerase chain reaction analysis of apoptosis-associated genes. " $\mathrm{P}<0.05$. AMPK, 5'adenosine monophosphateactivated protein kinase; Bad, Bcl2-associated agonist of cell death; Bax, Bcl-2-associated X protein; Bcl2, B-cell lymphoma 2; c-IAP, cellular inhibitor of apoptosis protein 1; cle, cleaved; Mel, melatonin; STZ, streptozotocin; WT, wild-type.

epithelial desquamation, renal tubule degeneration and kidney glomerular basement membrane thickening (Fig. 3B-F). However, these renal histopathological alterations were diminished by melatonin treatment in an AMPK-dependent manner.

Notably, the preservation of renal structural integrity was closely associated with an improvement in kidney function. Renal function was assessed by determining blood urea nitrogen (BUN) and serum creatinine levels at the end of the experiment. Compared with the control WT mice, STZ increased the levels of BUN (Fig. 3G) and serum creatinine (Fig. 3H). However, melatonin application reduced the BUN and serum creatinine levels, potentially by restoring AMPK activity. Additionally, urinary albumin content and the albumin-to-creatinine ratio (ACR) were measured to determine the severity of diabetic nephropathy. As shown in Fig. 3I-J, compared with the control WT mice, STZ-treated mice exhibited significantly increased urinary albumin content level and ACR. However, melatonin administration significantly reduced the urinary albumin and ACR in the STZ-treated mice, whereas the protective effect of melatonin was inhibited by AMPK deletion.

Melatonin sustains glomerular survival by activating the AMPK/PGCal pathway in vitro. Diabetic renal fibrosis is involved in glomerular apoptosis; therefore, it was of interest to investigate whether melatonin may suppress hyperglycemia-induced glomerular damage via AMPK. Firstly, cellular viability and apoptosis were analyzed by MTT assay and TUNEL staining, respectively. Compared with the normal group, high glucose treatment significantly reduced cellular viability (Fig. 4A) and significantly increased apoptosis of HBZY-1 cells (Fig. 4B and C). However, melatonin treatment sustained cellular viability and suppressed 

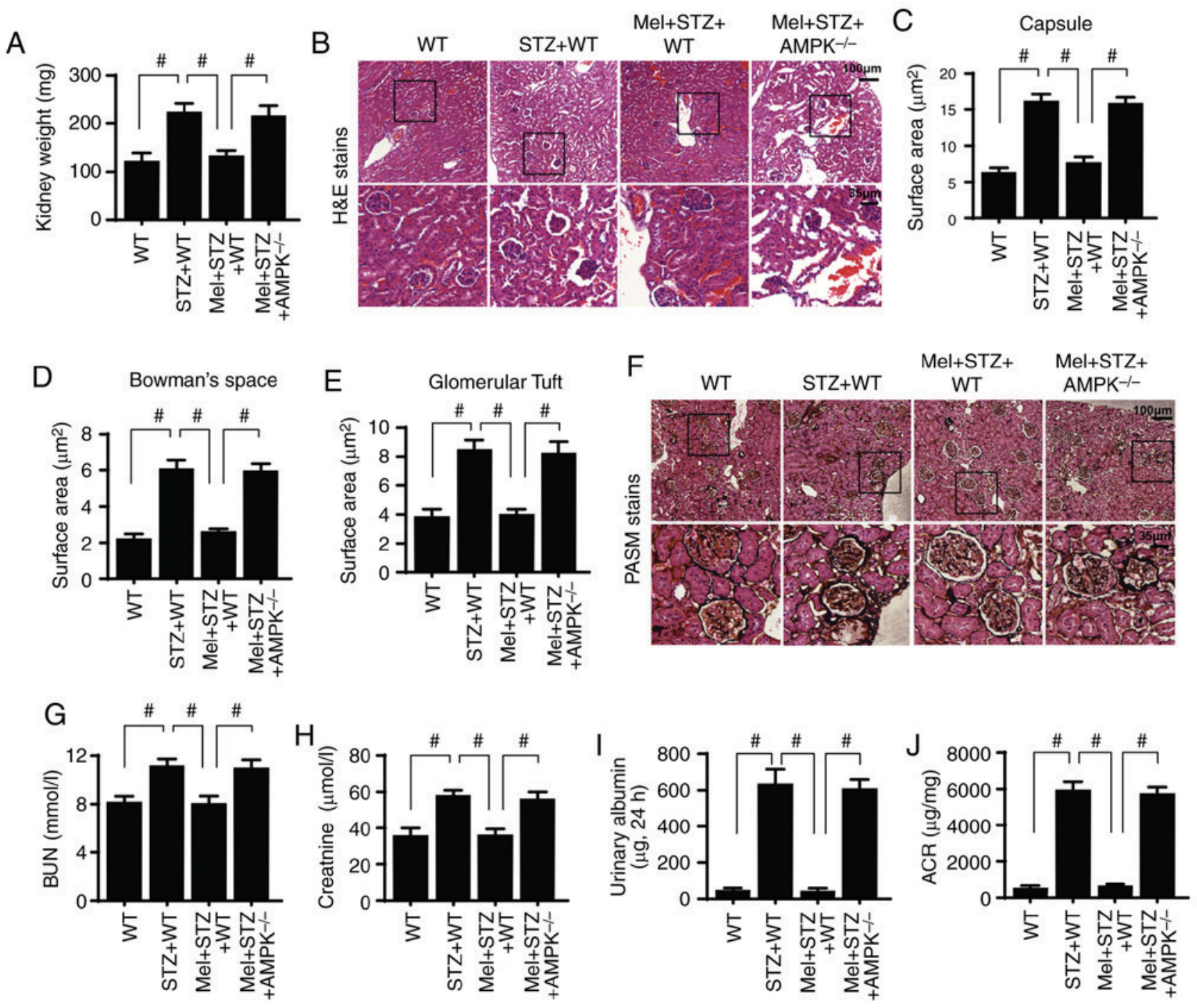

Figure 3. Melatonin attenuates diabetic renal injury in an AMPK-dependent manner. (A) Kidney weights of STZ-treated mice in the presence or absence of melatonin. (B) H\&E staining of kidney sections. Surface area of the (C) Bowman's capsule, (D) Bowman's space and (E) glomerular tuft. (F) PASM staining of kidney tissues. (G) BUN, (H) serum creatinine, (I) urinary albumin and (J) ACR levels were measured. " $\mathrm{P}<0.05$. ACR, albumin-to-creatinine ratio; AMPK, 5'adenosine monophosphate-activated protein kinase; BUN, blood urea nitrogen; H\&E, hematoxylin and eosin; Mel, melatonin; PASM, periodic acid Schiff-methenamine; STZ, streptozotocin; WT, wild-type.

hyperglycemia-induced apoptosis, whereas silencing AMPK resulted in a loss of these effects.

It has previously been suggested that PGC $\alpha 1$ is the downstream mediator of AMPK, and activated PGC $\alpha 1$ favors cellular survival via inhibition of the mitochondrial apoptosis pathway (49). Therefore, PGC $\alpha 1$ activation may be required for the kidney protection exerted by melatonin/AMPK pathways. The present results demonstrated that PGC $\alpha 1$ expression was reduced by high glucose-induced stress and was reversed back to normal levels with melatonin treatment (Fig. 4D-F). However, silencing AMPK abolished PGC $\alpha 1$ expression, thus suggesting that PGC $\alpha 1$ was activated by melatonin/AMPK. To understand the role of PGC $\alpha 1$ in glomerular survival, PGC $\alpha 1$ expression was knocked down in melatonin-treated HBZY-1 cells (Fig. 4D-F). Following inhibition of PGC $\alpha 1$ expression, the protective role of melatonin on cellular apoptosis was inhibited, as indicated by the LDH release assay (Fig. 4G), which was similar to the results obtained in AMPK-silenced cells. In addition, these findings were further supported by the caspase-3 activity assay (Fig. 4H). Therefore, these data indicated that the AMPK/PGC $\alpha 1$ pathway was necessary for melatonin-induced glomerular survival under high glucose treatment.

Melatonin suppresses mitochondrial apoptosis via the $A M P K / P G C \alpha 1$ pathway in vitro. Considering the well-established role of mitochondrial damage in glomerular apoptosis and diabetic renal fibrosis, the role of the melatonin/AMPK/PGC $\alpha 1$ pathway in mitochondrial homeostasis was investigated. Mitochondrial apoptosis is activated by ROS overproduction, membrane potential collapse, pro-apoptotic factor release and caspase family activation $(4,37)$. Compared with the normal group, high glucose evoked excessive ROS production (Fig. 5A and B), which was accompanied by a decline in mitochondrial potential (Fig. 5C and D). In addition, the mitochondrial pro-apoptotic protein cyt-c was rapidly released into the nucleus (Fig. 5E and F), in turn leading to an upregulation of other pro-apoptotic proteins, including caspase-3, caspase- 9 and 

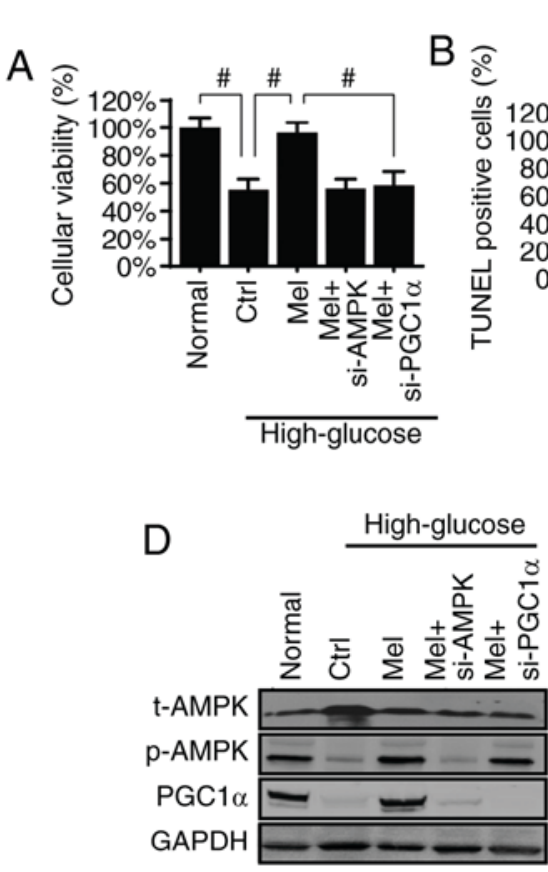
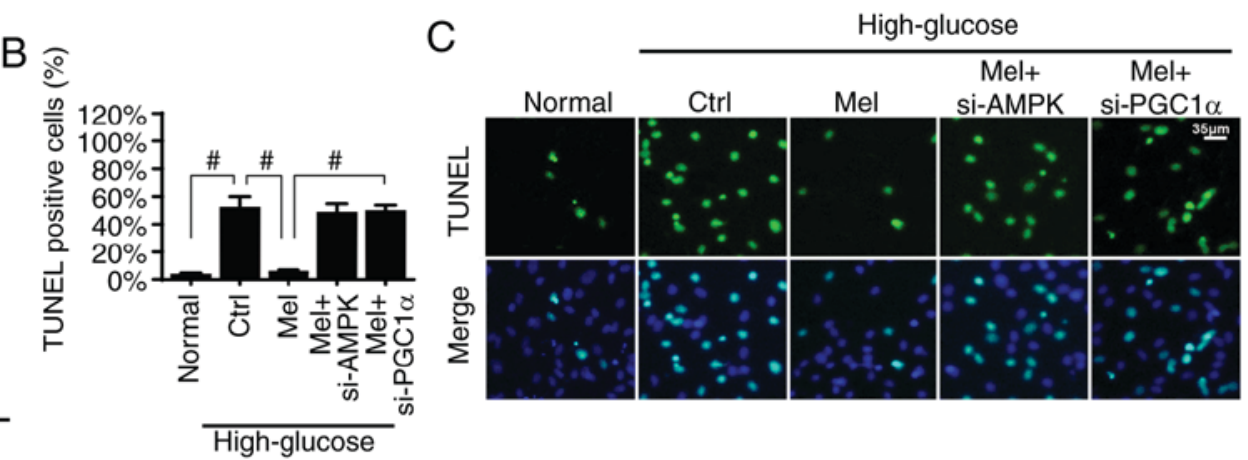

High-glucose
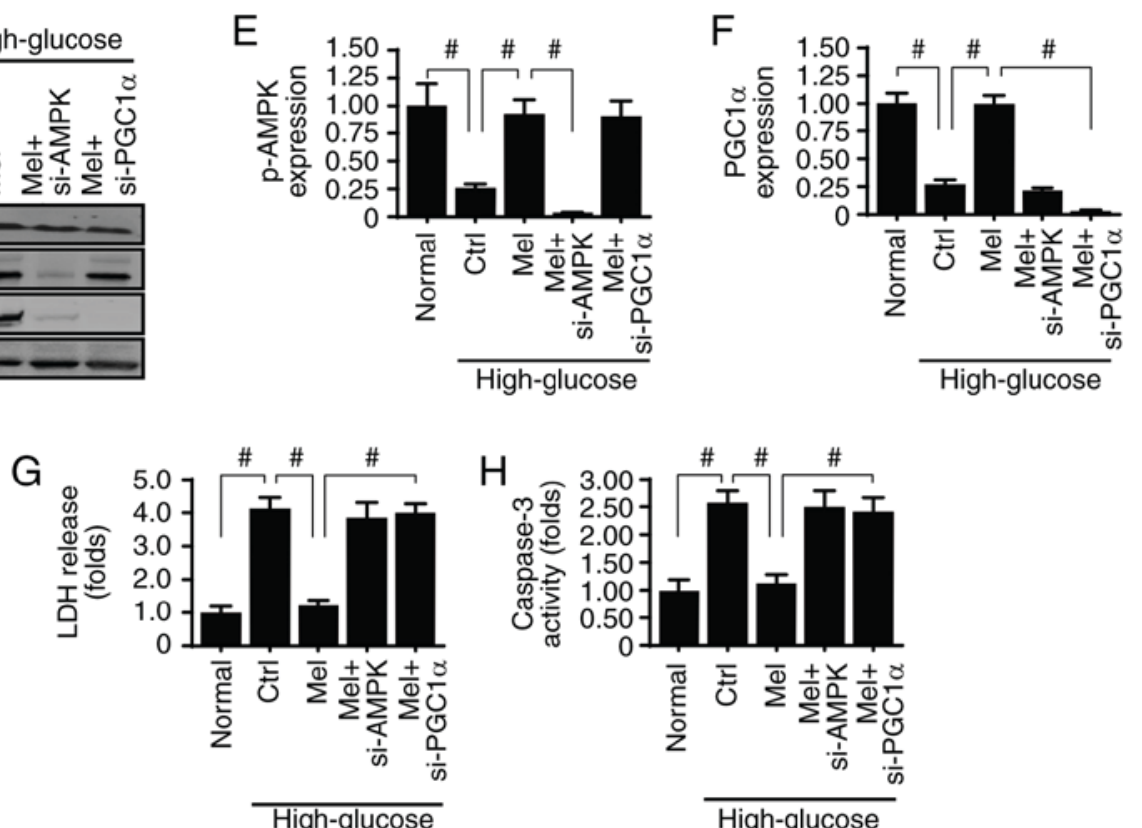

Figure 4. Melatonin sustains glomerular survival by activating the AMPK/PGCl $\alpha$ pathway. siRNAs were used to suppress melatonin-activated AMPK and PGCl $\alpha$ in the context of high glucose stress. (A) Viability of HBZY-1 cells was measured by MTT assay. (B and C) Quantification of apoptotic cells was conducted using the TUNEL assay. (D-F) Western blot analysis of AMPK and PGCl $\alpha$ expression. (G) Measurement of cellular death using the LDH release assay. (H) Measurement of caspase-3 activity to evaluate cellular apoptosis. ${ }^{~} \mathrm{P}<0.05$. AMPK, 5'adenosine monophosphate-activated protein kinase; Ctrl, control; LDH, lactate dehydrogenase; Mel, melatonin; p, phosphorylated; PGC1 $\alpha$, peroxisome proliferator-activated receptor $\gamma$ coactivator 1- $\alpha$; si, small interfering; t, total; TUNEL, terminal deoxynucleotidyl-transferase-mediated dUTP nick end labeling.

Bax (Fig. 5G-J). Conversely, the mitochondrial anti-apoptotic factor $\mathrm{Bcl} 2$ was downregulated under high glucose-induced stress (Fig. 5G and K). These results indicated that mitochondrial apoptosis was activated by hyperglycemia. Conversely, melatonin treatment neutralized ROS generation (Fig. 5A and B), maintained mitochondrial potential (Fig. 5C and D), suppressed cyt-c release (Fig. 5E and F), and corrected the imbalance between mitochondrial anti- and pro-apoptotic proteins (Fig. 5G and K). Notably, silencing of AMPK or PGC $\alpha 1$ attenuated the beneficial effects of melatonin on mitochondrial damage. Therefore, these data indicated that melatonin sustained mitochondrial homeostasis by activating the AMPK/PGC $\alpha 1$ pathway, thereby protecting the glomerulus against glucotoxicity.

Melatonin activates the AMPK/PGCal pathway to preserve mitochondrial function and structure in vitro. Besides mitochondrial apoptosis, mitochondrial function and structure are essential for cellular viability $(10,50)$. The primary function of mitochondria is to supply sufficient energy for cellular metabolism. The ATP content was reduced by high glucose treatment
(Fig. 6A) and was reversed to normal levels in response to melatonin administration via activation of the AMPK/PGC $\alpha 1$ pathway. Mechanistically, mitochondria produce ATP via the respiratory electron-transport chain (ETC) $(38,51)$. The activity of ETC was decreased by high glucose treatment (Fig. 6B-D) and was reversed to normal levels with melatonin application. However, inhibition of the AMPK/PGC $\alpha 1$ pathway abolished the protective effects of melatonin on ETC activity.

Renal functional impairment is closely associated with structural damage. Compared with the normal group, high glucose medium promoted the opening of mPTP, an early indicator of mitochondrial damage. Notably, melatonin was able to activate the AMPK/PGC $\alpha 1$ pathway and thereby modify the mPTP opening rate (Fig. 6E). At the molecular level, mPTP opening is regulated by cyclophilin $\mathrm{D}$, whereby p-cyclophilin $\mathrm{D}$ enhances mPTP opening $(52,53)$. Based on this, the present study investigated whether melatonin and the AMPK/PGC $\alpha 1$ pathway controls mPTP opening via cyclophilin D. As shown in Fig. 6F and G, cyclophilin D phosphorylation was increased in response to high glucose treatment and was decreased 
A

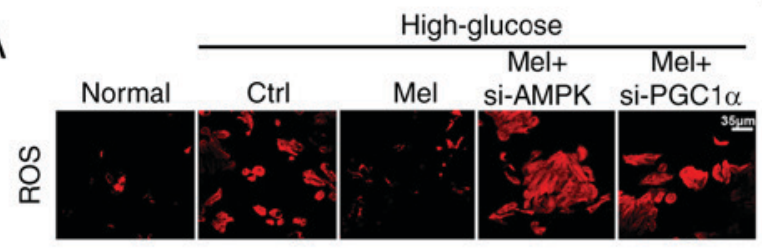

B

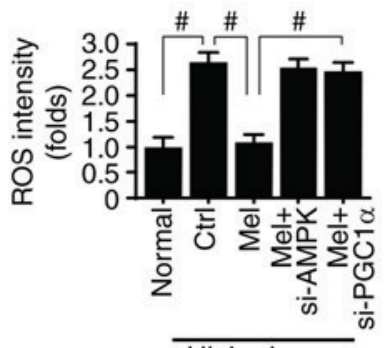

High-glucose
C

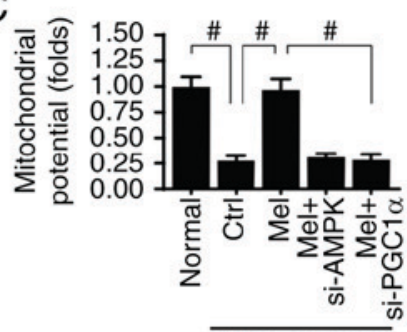

High-glucose

High-glucose

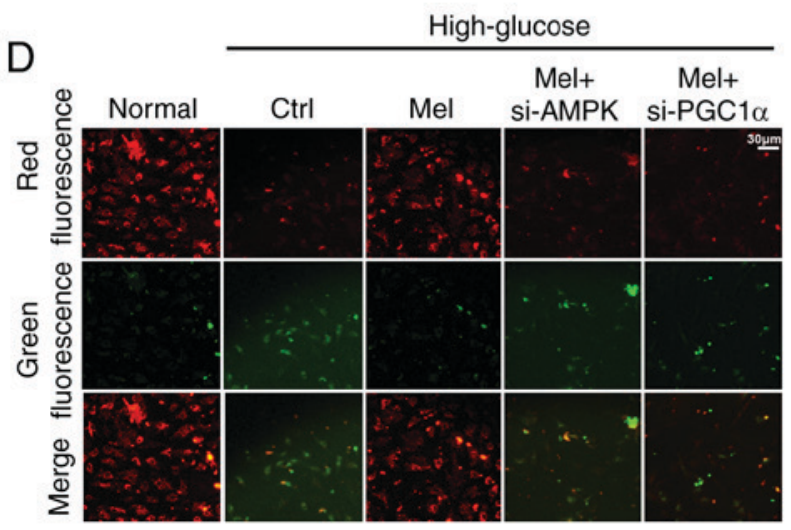

$\mathrm{F}$ 竞
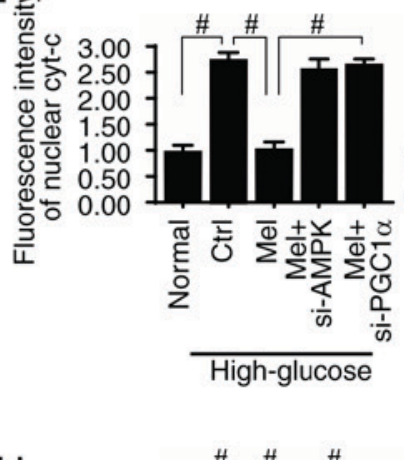

High-glucose

G

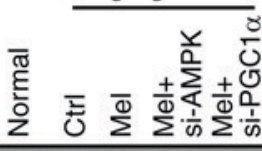

Pro.caspase-3

Cle.caspase-3

Caspase-9

$\mathrm{Bax}--\div-$

$\mathrm{Bcl} 2$

GAPDH

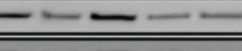

$\mathrm{H}$
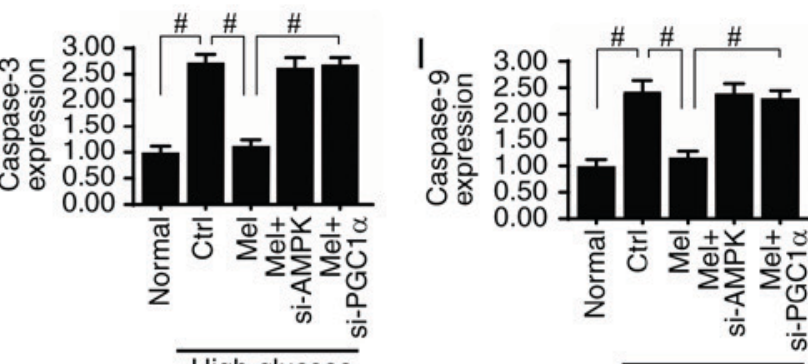

High-glucose

High-glucose

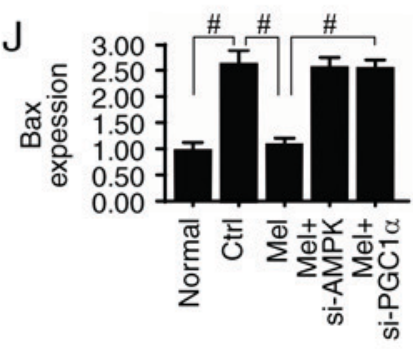

High-glucose

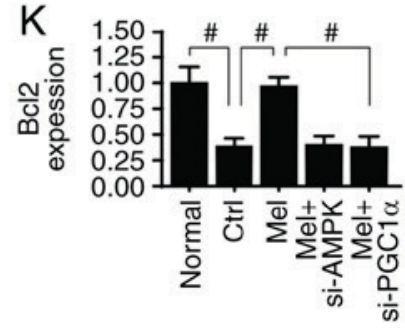

High-glucose

Figure 5. Melatonin suppresses high glucose-induced mitochondrial apoptosis by upregulating the AMPK/PGC1 $\alpha$ signaling pathway. (A and B) Dihydroethidium staining of cellular ROS. (C and D) JC-1 staining was used to detect changes in mitochondrial membrane potential. Green fluorescence indicates low mitochondrial membrane potential and red fluorescence represents high mitochondrial membrane potential. (E and F) Immunofluorescence staining of cyt-c and analysis of nuclear translocation. (G-K) Western blot analysis of apoptosis-associated proteins in HBZY-1 cells. ${ }^{\# P}<0.05$. AMPK, 5'adenosine monophosphate-activated protein kinase; Bax, Bcl-2-associated X protein; Bcl2, B-cell lymphoma 2; cle, cleaved; Ctrl, control; Cyt-c, cytochrome $c$; Mel, melatonin; PGC1 $\alpha$, peroxisome proliferator-activated receptor $\gamma$ coactivator $1-\alpha$; ROS, reactive oxygen species; si, small interfering.

with melatonin supplementation. Knockdown of AMPK and PGC $\alpha 1$ upregulated p-cyclophilin D expression, confirming that melatonin may maintain mitochondrial structure and function via the AMPK/PGC $\alpha 1$ pathway.

\section{Discussion}

The prevalence of diabetes continues to rise and is a global health burden. Diabetic renal fibrosis affects a significant percentage of patients with diabetes and contributes greatly to mortality. Previous studies have demonstrated that mitochondrial injury serves a primary role in the development of diabetic renal fibrosis $(54,55)$, which is line with the findings of the present study. In addition, the present study further explored the mechanism by which hyperglycemia evoked mitochondrial damage. Based on the findings, it may be hypothesized that AMPK downregulation and PGC $\alpha 1$ inactivation are responsible for the hyperglycemia-induced mitochondrial dysfunction and cellular apoptosis. Mechanistically, suppression of the AMPK/PGC $\alpha 1$ pathway promoted mitochondrial 


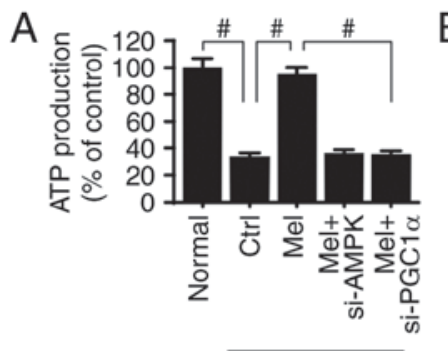

High-glucose

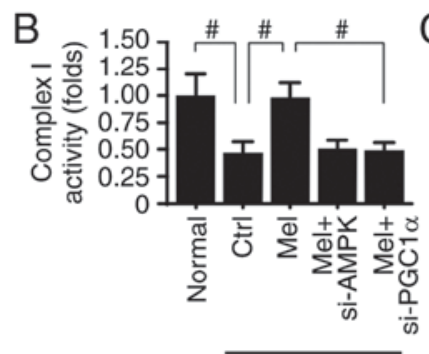

High-glucose

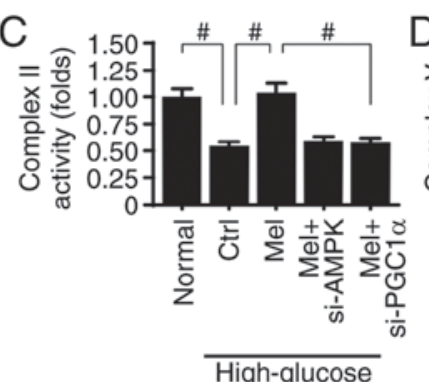

$\overline{\text { High-glucose }}$

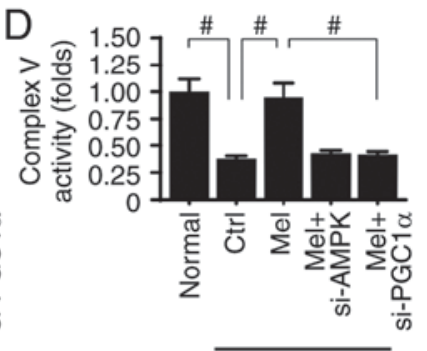

High-glucose
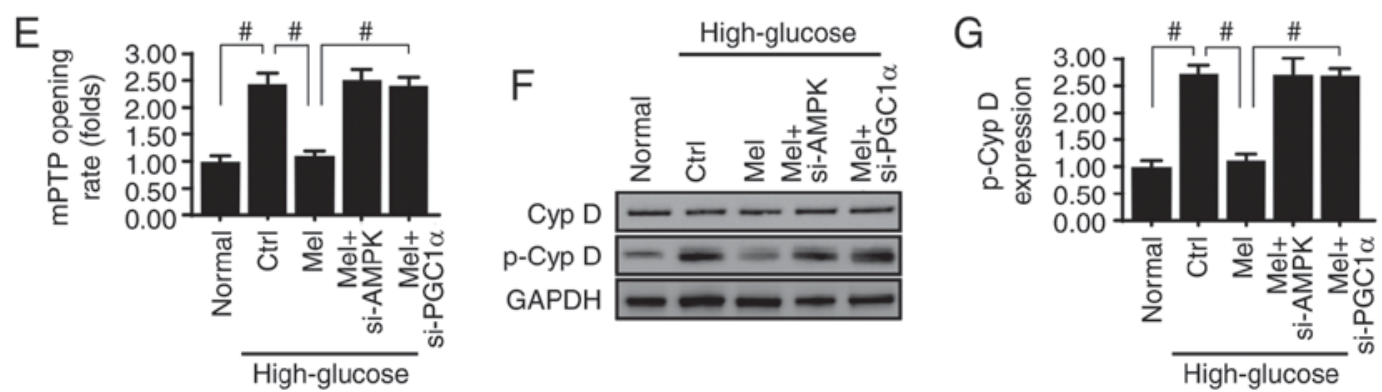

Figure 6. Melatonin activates the AMPK/PGC1 $\alpha$ signaling pathway to sustain mitochondrial homeostasis. (A) ATP production was detected using a Luciferase-based ATP Assay. (B-D) Analysis of the mitochondrial respiratory complex activity. (E) mPTP opening rate was detected using tetramethylrhodamine ethyl ester fluorescence. ( $\mathrm{F}$ and $\mathrm{G}$ ) Western blot analysis of Cyp D phosphorylation. ${ }^{~} \mathrm{P}<0.05$. AMPK, 5' adenosine monophosphate-activated protein kinase; ATP, adenosine 5'-triphosphate; Ctrl, control; Cyp D, cyclophilin D; Mel, melatonin; mptp, mitochondrial permeability transition pore; PGC1 $\alpha$, peroxisome proliferator-activated receptor $\gamma$ coactivator 1- $\alpha$; p, phosphorylated; si, small interfering.

oxidative stress, triggered mitochondrial potential collapse, enhanced pro-apoptotic cyt-c leakage into the nucleus and finally initiated caspase-9-induced mitochondrial apoptosis in the glomerulus. In addition, the inactive AMPK/PGC $\alpha 1$ pathway was instrumental to renal dysfunction and kidney fibrosis. Therefore, these findings comprehensively describe the causal relationship between the AMPK/PGC $\alpha 1$ pathway and diabetic renal fibrosis, which may provide a potential target to retard and/or reverse the progression of renal fibrosis induced by glucotoxicity.

Based on the present findings, mitochondrial damage was the initial upstream signal for glomerular injury and subsequent renal fibrosis. This conclusion was in agreement with previous studies $(56,57)$. Hyperglycemia has been reported to induce mitochondrial damage via numerous mechanisms. Hyperglycemia causes mitochondrial fragmentation and reduces mitochondrial ATP production. Additionally, high glucose stimulation evokes an excessive NLR family pyrin domain containing 3 -associated inflammatory response, leading to mitochondrial dysfunction and oxidative stress (58). Pierelli et al (59) demonstrated that hyperglycemia-induced downregulation of uncoupling protein 2 expression impairs mitochondrial membrane potential and induces energy metabolism disorder. Besides, suppression of the mitochondrial tricarboxylic acid cycle due to chronic high glucose injury causes energy undersupply (60) and results in abnormal lipid metabolism. Similar to these reports, the present study demonstrated that high glucose induced excessive ROS production and impaired the glomerular anti-oxidative system, resulting in opening of the mPTP. Subsequently, hydrogen can be liberated, which accounts for the reduction in mitochondrial potential and disruption of ATP production $(61,62)$. The mPTP opening can also facilitate leakage of the pro-apoptotic factor cyt-c into the cytoplasm or nucleus, where it activates the caspase-9-associated mitochondrial apoptotic pathway $(63,64)$. Based on these data, the potential processes underlying mitochondrial injury induced by glucotoxicity were determined and these findings may further extend our understanding of hyperglycemia-mediated mitochondrial damage.

Additionally, melatonin was demonstrated to be an effective approach to protect mitochondria against glucotoxicity. Notably, melatonin may directly reduce diabetic renal damage by sustaining mitochondrial homeostasis. This notion has been supported by ample evidence, which has documented the role of melatonin in mitochondrial protection $(27,64,65)$. Melatonin suppresses excessive mitochondria fission (19), reverses protective mitophagy (24), scavenges mitochondrial ROS (66), alleviates mitochondrial calcium overload $(18,67)$, sustains mitochondrial energy metabolism (68), modifies mitochondrial sirtuin protein (69) and blocks the caspase-9 apoptosis pathway $(61,70)$. These findings are in line with the results in the present study. However, the key finding in the present study is that the AMPK/PGC1 $\alpha$ pathway was required for melatonin-mediated mitochondrial protection, glomerular survival and renal fibrosis inhibition. Similarly, recent studies have reported that melatonin can activate AMPK and protect cardiac endothelial cells against reperfusion injury $(25,26)$. In addition, it has been demonstrated that genetic ablation of AMPK negated the protective role of AMPK on endothelial survival and mitochondrial protection. Other studies have also revealed that AMPK activation by drugs, including liraglutide and empagliflozin, inhibits the progression of diabetes $(6,71)$. Therefore, these findings suggested that influencing the stability of AMPK may be a potential therapeutic tool to limit diabetes development and progression. 
In conclusion, through loss-of-function assays in vivo and in vitro, the present study confirmed that diabetic renal fibrosis was associated with mitochondrial damage via a decline in AMPK/PGC1 $\alpha$ activity. In addition, melatonin administration was capable of rescuing the AMPK/PGC1 $\alpha$ pathway, preserving mitochondrial homeostasis and reducing diabetic renal fibrosis.

\section{Acknowledgements}

Not applicable.

\section{Funding}

No funding was received.

\section{Availability of data and materials}

All data generated or analyzed during this study are included in this published article.

\section{Authors' contributions}

JL conceived the project. STY, XYM, ZYG and YHL performed the experiments. YHS and NL collected and analyzed the data. All authors participated in discussing and revising the manuscript.

\section{Ethics approval and consent to participate}

The animal study was performed in accordance with The Declaration of Helsinki. All experimental protocols were approved by the Ethics Committee of Chinese PLA General Hospital (Beijing, China; ethics reference no. 2017013).

\section{Patient consent for publication}

Not applicable.

\section{Competing interests}

The authors declare that they have no competing interests.

\section{References}

1. Hung YC, Lin YC, Hsieh HM, Huang CJ and Chiu HC: Impact of non-apnea sleep disorders on diabetic control and metabolic outcome-A population-based cohort study. Gen Hosp Psychiatry 52: 1-7, 2018.

2. Zaman SB, Karim MA, Hossain N, Al Kibria GM and Islam SMS: Plasma triglycerides as a risk factor for chronic kidney disease in type 2 diabetes mellitus: Evidence from the northeastern of Thailand. Diabetes Res Clin Pract 138: 238-245, 2018.

3. Hu S, Gao Y, Zhou H, Kong F, Xiao F, Zhou P and Chen Y: New insight into mitochondrial changes in vascular endothelial cells irradiated by gamma ray. Int J Radiat Biol 93: 470-476, 2017.

4. Li R, Xin T, Li D, Wang C, Zhu H and Zhou H: Therapeutic effect of Sirtuin 3 on ameliorating nonalcoholic fatty liver disease: The role of the ERK-CREB pathway and Bnip3-mediated mitophagy. Redox Biol 18: 229-243, 2018.

5. Zhou H, Wang J, Zhu P, Hu S and Ren J: Ripk3 regulates cardiac microvascular reperfusion injury: The role of IP3R-dependent calcium overload, XO-mediated oxidative stress and F-action/filopodia-based cellular migration. Cell Signal 45: 12-22, 2018
6. Zhou H, Yang J, Xin T, Li D, Guo J, Hu S, Zhou S, Zhang T, Zhang Y, Han T and Chen Y: Exendin-4 protects adipose-derived mesenchymal stem cells from apoptosis induced by hydrogen peroxide through the PI3K/Akt-Sfrp2 pathways. Free Radic Biol Med 77: 363-375, 2014.

7. Zhang Y, Zhou H, Wu W, Shi C, Hu S, Yin T, Ma Q, Han T, Zhang Y, Tian F and Chen Y: Liraglutide protects cardiac microvascular endothelial cells against hypoxia/reoxygenation injury through the suppression of the SR-Ca(2+)-XO-ROS axis via activation of the GLP-1R/PI3K/Akt/survivin pathways. Free Radic Biol Med 95: 278-292, 2016.

8. Zhou H, Li D, Shi C, Xin T, Yang J, Zhou Y, Hu S, Tian F, Wang J and Chen Y: Effects of Exendin-4 on bone marrow mesenchymal stem cell proliferation, migration and apoptosis in vitro. Sci Rep 5: 12898, 2015.

9. Zhou H, Wang J, Zhu P, Zhu H, Toan S, Hu S, Ren J and Chen Y: NR4A1 aggravates the cardiac microvascular ischemia reperfusion injury through suppressing FUNDC1-mediated mitophagy and promoting Mff-required mitochondrial fission by CK $2 \alpha$. Basic Res Cardiol 113: 23, 2018.

10. Randriamboavonjy V, Kyselova A, Elgheznawy A, Zukunft S, Wittig I and Fleming I: Calpain 1 cleaves and inactivates prostacyclin synthase in mesenteric arteries from diabetic mice. Basic Res Cardiol 112: 10, 2017.

11. Ronchi C, Torre E, Rizzetto R, Bernardi J, Rocchetti M and Zaza A: Late sodium current and intracellular ionic homeostasis in acute ischemia. Basic Res Cardiol 112: 12, 2017.

12. Zhou H, Ma Q, Zhu P, Ren J, Reiter RJ and Chen Y: Protective role of melatonin in cardiac ischemia-reperfusion injury: From pathogenesis to targeted therapy. J Pineal Res: 64, 2018. doi: 10.1111/jpi.12471.

13. Motawi TK, Ahmed SA, A Hamed M, El-Maraghy SA and M Aziz W: Melatonin and/or rowatinex attenuate streptozotocin-induced diabetic renal injury in rats. J Biomed Res: Nov 1,2017 (Epub ahead of print).

14. Hrenak J, Paulis L, Repova K, Aziriova S, Nagtegaal EJ, Reiter RJ and Simko F: Melatonin and renal protection: Novel perspectives from animal experiments and human studies (review). Curr Pharm Des 21: 936-949, 2015.

15. Garcia-Nino WR, Correa F, Rodriguez-Barrena JI, León-Contreras JC, Buelna-Chontal M, Soria-Castro E, Hernández-Pando R, Pedraza-Chaverri J and Zazueta C: Cardioprotective kinase signaling to subsarcolemmal and interfibrillar mitochondria is mediated by caveolar structures. Basic Res Cardiol 112: 15, 2017.

16. Li Y, Wu H, Liu N, Cao X, Yang Z, Lu B, Hu R, Wang X and Wen J: Melatonin exerts an inhibitory effect on insulin gene transcription via MTNR1B and the downstream Raf1/ERK signaling pathway. Int J Mol Med 41: 955-961, 2018.

17. Yu LM, Di WC, Dong X, Li Z, Zhang Y, Xue XD, Xu YL, Zhang J, Xiao X, Han JS, et al: Melatonin protects diabetic heart against ischemia-reperfusion injury, role of membrane receptor-dependent cGMP-PKG activation. Biochim Biophys Acta 1864: 563-578, 2018

18. Zhou H, Du W, Li Y, Shi C, Hu N, Ma S, Wang W and Ren J: Effects of melatonin on fatty liver disease: The role of NR4A1/DNA-PKcs/p53 pathway, mitochondrial fission, and mitophagy. J Pineal Res: 64, 2018. doi: 10.1111/jpi.12450.

19. Zhu H, Jin Q, Li Y, Ma Q, Wang J, Li D, Zhou H and Chen Y: Melatonin protected cardiac microvascular endothelial cells against oxidative stress injury via suppression of IP3R-[Ca $\left.{ }^{2+}\right]$ c/VDAC- $\left[\mathrm{Ca}^{2+}\right] \mathrm{m}$ axis by activation of MAPK/ERK signaling pathway. Cell Stress Chaperones 23: 101-113, 2018.

20. Zhou H, Wang S, Zhu P, Hu S, Chen Y and Ren J: Empagliflozin rescues diabetic myocardial microvascular injury via AMPK-mediated inhibition of mitochondrial fission. Redox Biol 15: 335-346, 2018.

21. Torres-Quesada O, Mayrhofer JE and Stefan E: The many faces of compartmentalized PKA signalosomes. Cell Signal 37: 1-11, 2017.

22. Jovancevic N, Dendorfer A, Matzkies M, Kovarova M, Heckmann JC, Osterloh M, Boehm M, Weber L, Nguemo F, Semmler J, et al: Medium-chain fatty acids modulate myocardial function via a cardiac odorant receptor. Basic Res Cardiol 112: $13,2017$.

23. Alghanem AF, Wilkinson EL, Emmett MS, Aljasir MA, Holmes K, Rothermel BA, Simms VA, Heath VL and Cross MJ: RCAN1.4 regulates VEGFR-2 internalisation, cell polarity and migration in human microvascular endothelial cells. Angiogenesis 20: 341-358, 2017. 
24. Zhou H, Zhu P, Wang J, Zhu H, Ren J and Chen Y: Pathogenesis of cardiac ischemia reperfusion injury is associated with CK2 $\alpha$-disturbed mitochondrial homeostasis via suppression of FUNDC1-related mitophagy. Cell Death Differ 25: 1080-1093, 2018.

25. Zhou H, Zhang Y, Hu S, Shi C, Zhu P, Ma Q, Jin Q, Cao F, Tian F and Chen Y: Melatonin protects cardiac microvasculature against ischemia/reperfusion injury via suppression of mitochondrial fiss ion-VDAC1-HK2-mPTP-mitophagy axis. J Pineal Res: 63, 2017. doi: 10.1111/jpi.12413.

26. Zhou H, Li D, Zhu P, Hu S, Hu N, Ma S, Zhang Y, Han T, Ren J, Cao F and Chen Y: Melatonin suppresses platelet activation and function against cardiac ischemia/reperfusion injury via PPAR $\gamma /$ FUNDC1/mitophagy pathways. J Pineal Res: 63, 2017. doi: 10.1111/jpi.12438.

27. Oanh NTK, Park YY and Cho H: Mitochondria elongation is mediated through SIRT1-mediated MFN1 stabilization. Cell Signal 38: 67-75, 2017

28. Fuhrmann DC and Brüne B: Mitochondrial composition and function under the control of hypoxia. Redox Biol 12: 208-215, 2017

29. Zhai M, Li B, Duan W, Jing L, Zhang B, Zhang M, Yu L, Liu Z, Yu B, Ren K, et al: Melatonin ameliorates myocardial ischemia reperfusion injury through SIRT3-dependent regulation of oxidative stress and apoptosis. J Pineal Res: 63, 2017. doi: 10.1111/jpi.12419.

30. Zhang R, Sun Y, Liu Z, Jin W and Sun Y: Effects of melatonin on seedling growth, mineral nutrition, and nitrogen metabolism in cucumber under nitrate stress. J Pineal Res: 62, 2017. doi: 10.1111/jpi.12403

31. Couto JA, Ayturk UM, Konczyk DJ, Goss JA, Huang AY, Hann S, Reeve JL, Liang MG, Bischoff J, Warman ML and Greene AK: A somatic GNA11 mutation is associated with extremity capillary malformation and overgrowth. Angiogenesis 20: 303-306, 2017.

32. Griffiths HR, Gao D and Pararasa C: Redox regulation in metabolic programming and inflammation. Redox Biol 12: 50-57, 2017.

33. Tallman KA, Kim HH, Korade Z, Genaro-Mattos TC, Wages PA Liu W and Porter NA: Probes for protein adduction in cholestero biosynthesis disorders: Alkynyl lanosterol as a viable sterol precursor. Redox Biol 12: 182-190, 2017.

34. Shi C, Cai Y, Li Y, Li Y, Hu N, Ma S, Hu S, Zhu P, Wang W and Zhou H: Yap promotes hepatocellular carcinoma metastasis and mobilization via governing cofilin/F-actin/lamellipodium axis by regulation of JNK/Bnip3/SERCA/CaMKII pathways. Redox Biol 14: 59-71, 2018.

35. Zhou H, Yang J, Xin T, Zhang T, Hu S, Zhou S, Chen G and Chen Y: Exendin- 4 enhances the migration of adipose-derived stem cells to neonatal rat ventricular cardiomyocyte-derived conditioned medium via the phosphoinositide 3-kinase/Akt-stroma cell-derived factor- $1 \alpha / \mathrm{CXC}$ chemokine receptor 4 pathway. Mol Med Rep 11: 4063-4072, 2015.

36. Livak KJ and Schmittgen TD: Analysis of relative gene expression data using real-time quantitative PCR and the 2(-Delta Delta C(T)) method. Methods 25: 402-408, 2001

37. Salminen A, Kaarniranta K and Kauppinen A: Integrated stress response stimulates FGF21 expression: Systemic enhancer of longevity. Cell Signal 40: 10-21, 2017.

38. Sigala F, Efentakis P, Karageorgiadi D, Filis K, Zampas P, Iliodromitis EK, Zografos G, Papapetropoulos A and Andreadou I: Reciprocal regulation of eNOS, $\mathrm{H}_{2} \mathrm{~S}$ and CO-synthesizing enzymes in human atheroma: Correlation with plaque stability and effects of simvastatin. Redox Biol 12: 70-81, 2017.

39. Jin Q, Li R, Hu N, Xin T, Zhu P, Hu S, Ma S, Zhu H, Ren J and Zhou H: DUSP1 alleviates cardiac ischemia/reperfusion injury by suppressing the Mff-required mitochondrial fission and Bnip3-related mitophagy via the JNK pathways. Redox Biol 14: 576-587, 2018

40. Zhou H, Zhu P, Guo J, Hu N, Wang S, Li D, Hu S, Ren J, Cao F and Chen Y: Ripk3 induces mitochondrial apoptosis via inhibition of FUNDC1 mitophagy in cardiac IR injury. Redox Biol 13 498-507, 2017

41. Yu S, Wang X, Geng P, Tang X, Xiang L, Lu X, Li J, Ruan Z, Chen J, Xie G, et al: Melatonin regulates PARP1 to control the senescence-associated secretory phenotype (SASP) in human fetal lung fibroblast cells. J Pineal Res: 63, 2017. doi: 10.1111/jpi.12405

42. Lee $\mathrm{K}$ and Back $\mathrm{K}$ : Overexpression of rice serotonin $\mathrm{N}$-acetyltransferase 1 in transgenic rice plants confers resistance to cadmium and senescence and increases grain yield. J Pineal Res: 62, 2017. doi: 10.1111/jpi.12392.
43. Fukumoto M, Kondo K, Uni K, Ishiguro T, Hayashi M, Ueda S, Mori I, Niimi K, Tashiro F, Miyazaki S, et al: Tip-cell behavior is regulated by transcription factor FoxO1 under hypoxic conditions in developing mouse retinas. Angiogenesis 21: 203-214, 2018

44. Lee HJ, Jung YH, Choi GE, Ko SH, Lee SJ, Lee SH and Han HJ: BNIP3 induction by hypoxia stimulates FASN-dependent free fatty acid production enhancing therapeutic potential of umbilical cord blood-derived human mesenchymal stem cells. Redox Biol 13: 426-443, 2017.

45. Liu Z, Gan L, Luo D and Sun C: Melatonin promotes circadian rhythm-induced proliferation through Clock/histone deacetylase 3/c-Myc interaction in mouse adipose tissue. J Pineal Res: 62 , 2017. doi: 10.1111/jpi.12383.

46. Le Cras TD, Mobberley-Schuman PS, Broering M, Fei L, Trenor CC III and Adams DM: Angiopoietins as serum biomarkers for lymphatic anomalies. Angiogenesis 20: 163-173, 2017.

47. Vargas LA, Velasquez FC and Alvarez BV: Compensatory role of the $\mathrm{NBCn} 1$ sodium/bicarbonate cotransporter on $\mathrm{Ca}^{2+}$-induced mitochondrial swelling in hypertrophic hearts. Basic Res Cardiol 112: 14, 2017.

48. Pickard JM, Burke N, Davidson SM and Yellon DM: Intrinsic cardiac ganglia and acetylcholine are important in the mechanism of ischaemic preconditioning. Basic Res Cardiol 112: 11, 2017.

49. Brasacchio D, Alsop AE, Noori T, Lufti M, Iyer S, Simpson KJ, Bird PI, Kluck RM, Johnstone RW and Trapani JA: Epigenetic control of mitochondrial cell death through PACS1-mediated regulation of BAX/BAK oligomerization. Cell Death Differ 24: 961-970, 2017.

50. Kim EJ, Kim SH, Jin X, Jin X and Kim H: KCTD2, an adaptor of Cullin3 E3 ubiquitin ligase, suppresses gliomagenesis by destabilizing c-Myc. Cell Death Differ 24: 649-659, 2017

51. Zhou H, Hu S, Jin Q, Shi C, Zhang Y, Zhu P, Ma Q, Tian F and Chen Y: Mff-dependent mitochondrial fission contributes to the pathogenesis of cardiac microvasculature Ischemia/reperfusion injury via induction of mROS-mediated cardiolipin oxidation and HK2/VDAC1 Disassociation-involved mPTP opening. J Am Heart Assoc 6: pii: e005328, 2017.

52. Solomon H, Brauning B, Fainer I, Ben-Nissan G, Rabani S, Goldfinger N, Moscovitz O, Shakked Z, Rotter V and Sharon M: Post-translational regulation of p53 function through $20 \mathrm{~S}$ proteasome-mediated cleavage. Cell Death Differ 24: 2187-2198, 2017.

53. Morozzi G, Beccafico S, Bianchi R, Riuzzi F, Bellezza I, Giambanco I, Arcuri C, Minelli A and Donato R: Oxidative stress-induced S100B accumulation converts myoblasts into brown adipocytes via an $\mathrm{NF}-\kappa \mathrm{B} / \mathrm{YY} 1 / \mathrm{miR}-133$ axis and NF- $\kappa$ B/YY1/BMP-7 axis. Cell Death Differ 24: 2077-2088, 2017.

54. Zhou H, Li D, Zhu P, Ma Q, Toan S, Wang J, Hu S, Chen Y and Zhang Y: Inhibitory effect of melatonin on necroptosis via repressing the Ripk3-PGAM5-CypD-mPTP pathway attenuates cardiac microvascular ischemia-reperfusion injury. J Pineal Res: May 16, e12503, 2018 (Epub ahead of print).

55. Zhou H, Wang S, Hu S, Chen Y and Ren J: ER-mitochondria microdomains in cardiac Ischemia-reperfusion injury: A fresh perspective. Front Physiol 9: 755, 2018.

56. Zhang W, Tao A, Lan T, Cepinskas G, Kao R, Martin CM and Rui T: Carbon monoxide releasing molecule-3 improves myocardial function in mice with sepsis by inhibiting NLRP3 inflammasome activation in cardiac fibroblasts. Basic Res Cardiol 112: 16, 2017.

57. Banerjee K, Keasey MP, Razskazovskiy V, Visavadiya NP, Jia C and Hagg T: Reduced FAK-STAT3 signaling contributes to ER stress-induced mitochondrial dysfunction and death in endothelial cells. Cell Signal 36: 154-162, 2017.

58. Rovira-Llopis S, Apostolova N, Bañuls C, Muntané J, Rocha M and Victor VM: Mitochondria, the NLRP3 inflammasome, and sirtuins in type 2 diabetes: New therapeutic targets. Antioxid Redox Signal 29: 749-791, 2018.

59. Pierelli G, Stanzione R, Forte M, Migliarino S, Perelli M, Volpe $\mathrm{M}$ and Rubattu S: Uncoupling protein 2: A key player and a potential therapeutic target in vascular diseases. Oxid Med Cell Longev 2017: 7348372, 2017.

60. Rauckhorst AJ, Gray LR, Sheldon RD, Fu X, Pewa AD, Feddersen CR, Dupuy AJ, Gibson-Corley KN, Cox JE, Burgess SC and Taylor EB: The mitochondrial pyruvate carrier mediates high fat diet-induced increases in hepatic TCA cycle capacity. Mol Metab 6: 1468-1479, 2017. 
61. Dufour F, Rattier T, Shirley S, Picarda G, Constantinescu AA, Morlé A, Zakaria AB, Marcion G, Causse S, Szegezdi E, et al: N-glycosylation of mouse TRAIL-R and human TRAIL-R1 enhances TRAIL-induced death. Cell Death Differ 24: 500-510, 2017.

62. Gao Y, Xiao X, Zhang C, Yu W, Guo W, Zhang Z, Li Z, Feng X, Hao J, Zhang K, et al: Melatonin synergizes the chemotherapeutic effect of 5-fluorouracil in colon cancer by suppressing PI3K/AKT and NF-kB/iNOS signaling pathways. J Pineal Res: 62, 2017. doi: 10.1111/jpi.12380.

63. Daiber A, Oelze M, Steven S, Kroller-Schon S and Münzel T: Taking up the cudgels for the traditional reactive oxygen and nitrogen species detection assays and their use in the cardiovascular system. Redox Biol 12: 35-49, 2017.

64. Zhou H, Shi C, Hu S, Zhu H, Ren J and Chen Y: BI1 is associated with microvascular protection in cardiac ischemia reperfusion injury via repressing Syk-Nox2-Drp1-mitochondrial fission pathways. Angiogenesis 21: 599-615, 2018.

65. Zhou H, Yue Y, Wang J, Ma Q and Chen Y: Melatonin therapy for diabetic cardiomyopathy: A mechanism involving Syk-mitochondrial complex I-SERCA pathway. Cell Signal 47: $88-100,2018$
66. Mayo JC, Sainz RM, Gonzalez Menendez P, Cepas V, Tan DX and Reiter RJ: Melatonin and sirtuins: A 'not-so unexpected' relationship. J Pineal Res: 62, 2017. doi: 10.1111/jpi.12391.

67. Lee JH, Han YS and Lee SH: Potentiation of biological effects of mesenchymal stem cells in ischemic conditions by melatonin via upregulation of cellular prion protein expression. J Pineal Res 62: 2017. doi: 10.1111/jpi.12385.

68. Lee HY and Back K: Melatonin is required for $\mathrm{H}_{2} \mathrm{O}_{2}$ - and NO-mediated defense signaling through MAPKKK3 and OXI1 in Arabidopsis thaliana. J Pineal Res: 62, 2017. doi: 10.1111/jpi.12379.

69. Lee MS, Yin TC, Sung PH, Chiang JY, Sun CK and Yip HK: Melatonin enhances survival and preserves functional integrity of stem cells: A review. J Pineal Res: 62, 2017. doi: 10.1111/jpi.12372.

70. Tamura H, Kawamoto M, Sato S, Tamura I, Maekawa R, Taketani T, Aasada H, Takaki E, Nakai A, Reiter RJ and Sugino N: Long-term melatonin treatment delays ovarian aging. J Pineal Res: 62, 2017. doi: 10.1111/jpi.12381.

71. Ligeza J, Marona P, Gach N, Lipert B, Miekus K, Wilk W, Jaszczynski J, Stelmach A, Loboda A, Dulak J, et al: MCPIP1 contributes to clear cell renal cell carcinomas development. Angiogenesis 20: 325-340, 2017. 